\title{
Wireless Analytics for 3D Printed Objects
}

\author{
Vikram Iyer, Justin Chan, Ian Culhane, Jennifer Mankoff and Shyamnath Gollakota \\ University of Washington \\ \{vsiyer, jucha, iac2, jmankoff, gshyam\}@uw.edu
}
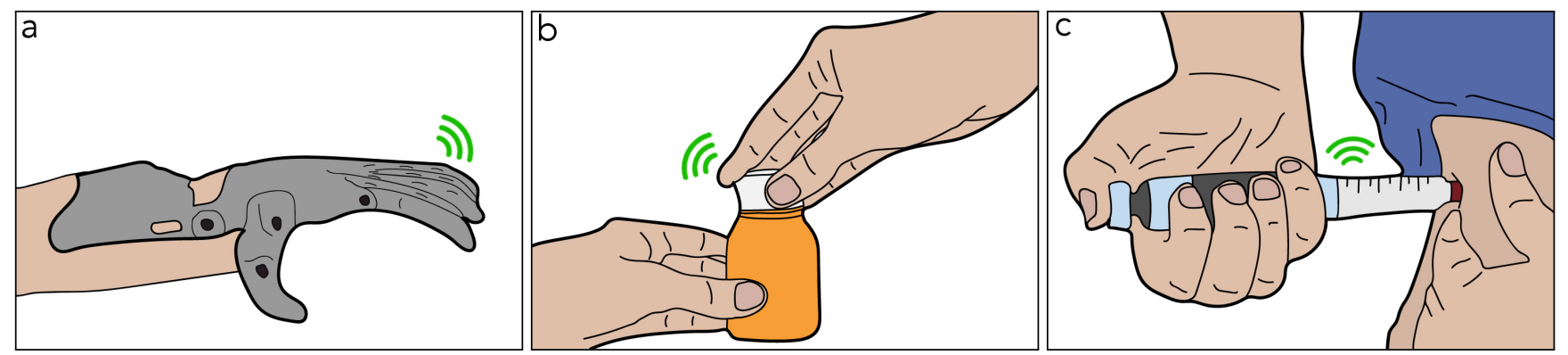

Figure 1: Example applications enabled by our design. (a) e-NABLE arm functioning as a wireless controller. (b) Pill bottle tracks when it is opened and closed. (c) Insulin pen stores usage data when it is outside the range of a wireless receiver.

\begin{abstract}
We present the first wireless physical analytics system for 3D printed objects using commonly available conductive plastic filaments. Our design can enable various data capture and wireless physical analytics capabilities for 3D printed objects, without the need for electronics. To achieve this goal, we make three key contributions: (1) demonstrate room scale backscatter communication and sensing using conductive plastic filaments, (2) introduce the first backscatter designs that detect a variety of bi-directional motions and support linear and rotational movements, and (3) enable data capture and storage for later retrieval when outside the range of the wireless coverage, using a ratchet and gear system. We validate our approach by wirelessly detecting the opening and closing of a pill bottle, capturing the joint angles of a 3D printed e-NABLE prosthetic hand, and an insulin pen that can store information to track its use outside the range of a wireless receiver.
\end{abstract}

\section{CCS Concepts}

Human-centered computing $\rightarrow$ Interaction devices; Interactive systems and tools;

\section{Author Keywords}

3D printing; digital fabrication; backscatter; prosthetic hand

\section{INTRODUCTION}

The growth of the 3D printing community has led to the creation and sharing of many useful objects such as assistive

Permission to make digital or hard copies of all or part of this work for personal or classroom use is granted without fee provided that copies are not made or distributed for profit or commercial advantage and that copies bear this notice and the full citation on the first page. Copyrights for components of this work owned by others than the author(s) must be honored. Abstracting with credit is permitted. To copy otherwise, or republish, to post on servers or to redistribute to lists, requires prior specific permission and/or a fee. Request permissions from Permissions@ acm.org.

UIST' 18 , October 14 - 17, 2018, Berlin, Germany

(C) 2018 Copyright is held by the owner/author(s). Publication rights licensed to ACM. ACM ISBN 978-1-4503-5948-1/18/10 _.. \$15.00

https://doi.org/10.1145/3242587.3242639 technologies $[1,10]$ and other functional objects [35]. If sensing could be embedded in such objects, it could increase their utility by supporting interactivity, and enable capture of information such as use over time.

In particular, it would be ideal if sensors could be printed, intrinsic to the objects being created, that could easily track their use, as expressed through invocation of their mechanical function. In fact, a variety of mechanisms exist for 3D printing sensors, such as detecting changes in pressure [51], proximity [30], light [43] and touch [47]. Such 3D printed sensors can be used to construct interactions such as knobs and sliders, are easy to customize, and require little or no assembly. However, these past works require close proximity to some sort of digital device such as a mobile phone, and lacked an ability to collect historical data when away from that device.

Better support for stand-alone 3D printed objects whose use can be captured wirelessly would open the door for a variety of analytics applications. For example, wireless capture of actuation of an e-NABLE prosthetic could yield valuable analytics about the use of the device over time. Similarly, in the context of prescription drugs, capturing the opening and closing of a 3D printed pill bottle could help manage patients' adherence to their doctors' guidelines or even potential misuse. More importantly, the ability to store this captured information within the $3 \mathrm{D}$ printed object itself would provide a practical solution for highly mobile objects. For example, a diabetic patient may carry a 3D printed insulin pen and use it outdoors away from wireless receivers but could upload the stored information about usage, with the push of a button upon returning home back into wireless coverage.

While pure wireless sensing has been recently demonstrated at very short ranges with printed conductive plastics [24] sensed using backscatter communication, the range is limited to less than $0.5 \mathrm{~m}$ and sensing is limited to uni-directional motion. However, achieving true wireless analytics requires i) support 
for room-scale sensing, ii) a rich set of sensing capabilities that go beyond just uni-directional motion and iii) wireless analytics capabilities beyond a single room by supporting capture and storage of data outside of wireless coverage.

This paper introduces PrintedAnalytics which addresses the above challenges and demonstrates, to the best of our knowledge, the first wireless, circuitless, physical analytics and capture system for 3D printed objects using commonly available conductive plastic filaments. To this end, we make the following key technical contributions.

- Room-scale wireless sensing. We present a solution to achieve room-scale communication ranges for $3 \mathrm{D}$ printed objects. Specifically, we increase the wireless communication range of $3 \mathrm{D}$ printed objects by leveraging interference cancellation [12] to enable receivers that can detect the weak backscattered signals from $3 \mathrm{D}$ printed objects. We are able to achieve a communication range of $4 \mathrm{~m}$, without the need for placing a wireless receiver close to the $3 \mathrm{D}$ printed object.

- Enriched sensor designs. Sensing analytics about device use requires the basic ability to sense bi-directional motion. We present a compact bi-directional backscatter switch, which provides the basic ability necessary for sensing mechanical device analytics. Specifically, unlike prior designs [24], that can support backscatter with only uni-directional motion, our new design can support bi-directional motion and is more compact. This allows it to be easily integrated within existing 3D printed objects like prosthetic arms and pill bottles that require bi-directional motion.

- Anywhere analytics. Full support for analytics requires sensing use over time, even when an object is out of sensor range. We present a mechanical capture and data storage mechanism. Our ratchet and gear storage system can function outside the range of a wireless receiver to store physical analytics data. It communicates the stored information when it is back in the receiver's range, with the push of a button.

We validate our approach, by integrating our backscatter designs within existing CAD models. Specifically, we integrate our solution with an e-NABLE prosthetic arm that can wirelessly backscatter information across a room. Our system is able to capture the arm opening and closing motions at an angular granularity of $15^{\circ}$. Additionally, we demonstrate a pill bottle with an integrated mechanism to capture its opening and closing. Finally we design an insulin pen prototype using our ratchet mechanism to store a user's dosage history by measuring how far the syringe's plunger has been depressed as a proxy for the amount of insulin administered by the user.

\section{RELATED WORK}

The ability to capture the interaction and use of objects is a natural next step after creating such objects, and one that has been explored in multiple bodies of work. One approach to interacting with $3 \mathrm{D}$ printed objects is to embed circuits in them (e.g., [44]). However, electronic-based solutions are more complex to design and assemble and require mastery of hardware and software. Further, they are less customizable than fully 3D printed objects. Thus, electronics-free approaches have seen significant recent interest.
One category of electronics-free approaches simply encodes data in 3D printed objects, which can then be retrieved with a reader. Acoustic Barcodes [21] use structured patterns of physical notches that when swiped produce a characteristic sound that can be resolved to a set of bits. Acoustic Voxels [32] 3D prints acoustic filters that modulate audio waves to encode data in the transmission loss curve. AirCode [33] stores data beneath the surface of a 3D printed object using carefully designed air pockets that can be read using computational imaging techniques. Lamello [45] uses 3D cantilevers printed along user interaction paths to create acoustic signals that can be read by a contact microphone placed on the object. Infrastructs [56] uses TeraHertz imaging techniques to read static information encoded in the design of an object at fabrication time. Maglink [24] manipulates the polarity of magnetized printed material to encode static data including bit strings on 3D printed objects. These approaches, however, require a nearby (often are wired to) computer or mobile device and cannot achieve the physical analytics and tracking capabilities shown in this paper.

A more difficult (and more powerful) option is to embed printable sensing in fabricated objects. A number of solutions exist that depend on an attached circuit and/or computer (e.g., $[30,43,47,51])$. Less work has focused on circuitless, wireless sensing. As described in the introduction, pure wireless sensing has been demonstrated in printed Wi-Fi [24] under very limited range conditions. In addition to the range limitations, [24] has limited sensing capabilities, can only support unidirectional motion and cannot capture and store data outside of wireless coverage. Another approach for wireless sensing, using RFID tags, is explored by [48] which uses copper or other material to block their visibility to a receiver and demonstrate sensing up to a range of about $1.5 \mathrm{~m}$. While RFID tags are an alternative to enabling wireless sensing, prior RFID work has not demonstrated the wide variety of mechanical functions shown in our paper. This is easier with fully 3D printed solutions, such as [24]. Further, the RFID sensing approach did not propose any mechanism for data capture and storage when outside of the range of the RFID reader. While chipless RFID designs $[15,16,41]$ have been explored, they require custom fabrication processes not yet available on commercial off-the-shelf 3D printers. The ability to 3D print wireless sensing capabilities, as demonstrated in this paper, allows the design of sensors that can be used to capture interaction with 3D printed objects (e.g., the angular motion of the e-NABLE prosthetic arm or use of an insulin pen). Our paper can be thought of as designing the first chipless tags at Wi-Fi frequencies using commodity $3 \mathrm{D}$ printers and enabling various data capture and wireless physical analytics capabilities.

Another related research effort is in the field of printed electronics $[11,17]$ which is focused on making basic components like transistors and interconnects. However printed electronics faces fundamental material hurdles for operation at RF frequencies and we believe it will be quite some time before printed electronics will become a reality. Our approach which leverages mechanical designs shows a way to achieve this connectivity today as well as provides a framework for future 3D printer designs (vis-a-vis, miniaturization). 


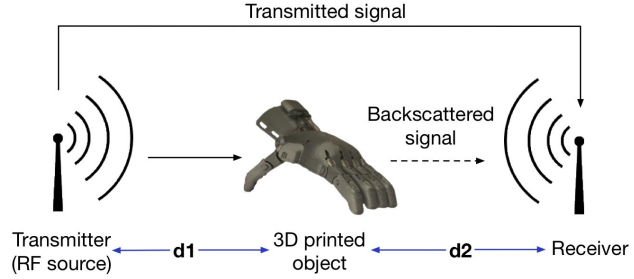

Figure 2: Printed backscatter design. The signal at the receiver is a combination of the transmitted signal from the RF source and the backscattered signal from the 3D printed object.

Finally, concurrent work [46] stores data about interaction with 3D printed objects using conductive fluids and reads them with touch screens. This is similar in spirit to the concept of anywhere analytics presented in our work but differs in that we introduce a mechanical capture and storage mechanism to store interaction and communicate the stored data using RF signals at a larger range, without requiring contact.

\section{ROOM-SCALE PRINTED BACKSCATTER}

We now briefly introduce backscatter and explain its suitability for 3D printing. We then present our first contribution, a novel printed backscatter approach for increasing transmit range.

\section{Primer on Backscatter Communication}

Backscatter communication systems transmit data by reflecting radio signals that are already on the wireless medium instead of generating their own. Most prior work on backscatter $[25,26,27,34,36,37,49,53,57]$ relies on electronics and digital logic to store and communicate data and hence cannot be $3 \mathrm{D}$ printed. However [24] demonstrated that it is possible to decouple a radio transmitter that generates those signals onto a device (e.g., a router) separate from the 3D printed object.

At a high level, a printed version of backscatter communication [24] has a regular radio transmitter (e.g., a router) that transmits radio signals on the wireless medium. The backscatter device reflects these radio signals, which are then decoded at another regular radio receiver. The backscatter device encodes data by modulating the power of its reflected signal by changing its radar cross-section.

While this modulation can be accomplished using electronics, the important insight of Printed Wi-Fi was that it can instead be managed mechanically, using a switch that can modulate the antenna's radar cross-section [24]. Printed Wi-Fi demonstrated that it is feasible to build backscatter devices using conductive plastic filaments with commodity 3D printers.

Specifically, a 3D printed dipole antenna that can radiate signals at Wi-Fi frequencies of $2.4 \mathrm{GHz}$ can be constructed using two narrow strips of conductive material separated by a gap. The switch connects the two halves when it is in contact with them and disconnects them otherwise, changing its reflective properties. Mechanical motion that occurs when a user interacts with the $3 \mathrm{D}$ printed objects can be translated to modulation of the switch connecting and disconnecting the two halves of the antenna. Since the reflection properties of an antenna depend on its length, the mechanical switch effectively

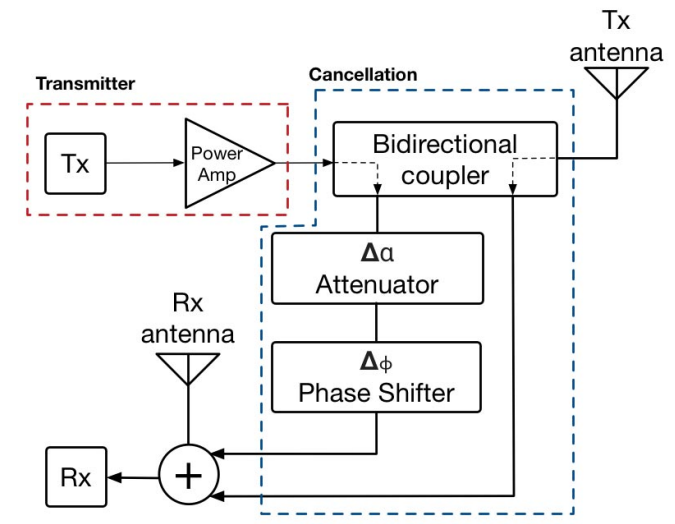

Figure 3: Block diagram of the full duplex radio setup.

changes the reflected signal from the 3D printed object, which can be decoded at a conventional wireless receiver.

Mathematically, we can express the effect of a backscatter switch toggling between two states using the following equation as a function of incident signal with power $P_{\text {incident }}$ :

$$
P_{\text {backscatter }}=P_{\text {incident }} \frac{\left|\Gamma_{1}^{*}-\Gamma_{2}^{*}\right|^{2}}{4}=P_{\text {incident }} \frac{|\Delta \Gamma|^{2}}{4}
$$

Here $\Gamma_{1}^{*}$ and $\Gamma_{2}^{*}$ represent the complex conjugates of the reflection coefficients, parameters describing how much of the signals are reflected in each state.

An advantage of backscatter communication is that it can be piggybacked on existing Wi-Fi traffic that already exists on the network, without affecting the Wi-Fi performance. This is because reflections are significantly weaker than that transmissions from Wi-Fi radios [26]. Furthermore, existing approaches demonstrate how to introduce additional Wi-Fi traffic to aid with wireless power as well as backscatter communication, while minimizing the impact on existing Wi-Fi networks using frequency hopping techniques [50]. Thus, the impact of backscatter on existing Wi-Fi traffic can be minimal.

\section{Increasing the Range of Printed Backscatter}

We first explain why the existing printed backscatter design [24] is of limited range. The wireless receiver receives a combination of both the transmitted signal from the RF source as well as the backscattered signal. Specifically, consider the deployment in Fig. 2 where the printed backscatter device is in between the RF source and the wireless receiver. Ignoring wireless multipath, the signal strength of the RF source at the wireless receiver can be written as, $P_{T X-R X}=\left(\frac{P_{T X} G_{T X}}{4 \pi\left(d_{1}+d_{2}\right)^{2}}\right)$. On the other hand, the power of the reflected signal, $P_{\text {backscatter }}$, is given by [6], $\left(\frac{P_{T X} G_{T X}}{4 \pi d_{1}^{2}}\right)\left(\frac{\lambda^{2} G_{\text {printed }}^{2}}{4 \pi} \frac{|\Delta \Gamma|^{2}}{4}\right)\left(\frac{1}{4 \pi d_{2}^{2}} \frac{\lambda^{2} G_{R X}}{4 \pi}\right)$. The first term of this equation represents the path loss of the transmitted signal sent with power $P_{T X}$ and antenna gain $G_{T X}$ at distance $d_{1}$ from the $3 \mathrm{D}$ printed object. The second term represents the backscattered signal as described above based on the change in reflection coefficient $\Delta \Gamma$ with additional parameters for its antenna gain $G_{\text {printed }}$ and the wavelength of the RF signal $\lambda$. 
The final term represents the second leg of path loss as the reflected signal goes to the receiver. To successfully detect a transmission from the 3D printed object we must then be able to differentiate that from the direct transmitted signal.

The preceding equations show that the backscatter signal attenuates as $d_{1}^{2} d_{2}^{2}$, while the direct signal from the transmitter to the receiver attenuates as $\left(d_{1}+d_{2}\right)^{2}$. Since the receiver receives the combination of both the transmitted signal from the signal source as well as the backscatter signal, the direct signal dominates the received signal, making it difficult for the receiver to decode the backscattered signals from the printed objects. Prior work [24] requires the wireless receiver to be next to the 3D printed objects. This ensures that $d_{2}$ is a small value $\varepsilon$ and thus $\left(\varepsilon+d_{1}\right)^{2}$ is close to $d_{1}^{2}$. As a result, the signal from the printed backscatter device has a comparable power to that directly from the RF source. This ensures that the wireless receiver can decode the backscattered signals in the presence of interference from the RF source. However, requiring the wireless receiver to be next to the printed object significantly limits the potential for 3D printed objects.

Our solution. To ensure that the backscattered signal can be decoded in the presence of interference from the direct transmission, we leverage the fact that the wireless receiver knows the exact signal transmitted by the RF source. Thus, it can effectively perform successive interference cancellation where it first subtracts the known signal from the RF source and then decodes the remaining backscatter signal.

Note that we leverage recent work on interference cancellation $[19,20]$ and full duplex $[8,12]$ for Wi-Fi radios, to achieve this cancellation. At a high level, say the RF source outputs a known signal, $x_{r e f}(t)$ on the wireless medium. As this signal propagates over the wireless channel from the transmitter to the receiver, it will experience some amplitude change $\alpha$ and phase change $\phi$. In order to cancel the direct path, we simply need to add its inverse which we can obtain by scaling and phase shifting the known signal, $x_{r e f}(t)$. The above cancellation can be performed either when the receiver is placed far away from the RF source or is next to it. In the first case, we can use interference cancellation techniques which estimate the channel from a known preamble and perform the above cancellation at the wireless receiver. In the second scenario, we effectively leverage full duplex radios which use a single antenna connected to a specialized RF component such as a circulator or bi-directional coupler which can provide a reference of the transmitter that is isolated from the received signal path. We implement this as shown in Fig 3. For our transmitter we use a USRP software radio and connect its output to a power splitter. We connect one output to a power amplifier to broadcast over the air and use the other as our reference signal. We first connect the reference signal to a variable attenuator RVA-33 [4] cascaded with a variable phase shifter. We then use a power combiner to sum this signal with the raw received signal from the antenna to produce the final output.

Considering we use an analog phase shifter and attenuator we could in theory match the transmitted signal exactly and achieve even excellent cancellation, however this is complicated by a variety of factors. In addition to the electronic noise

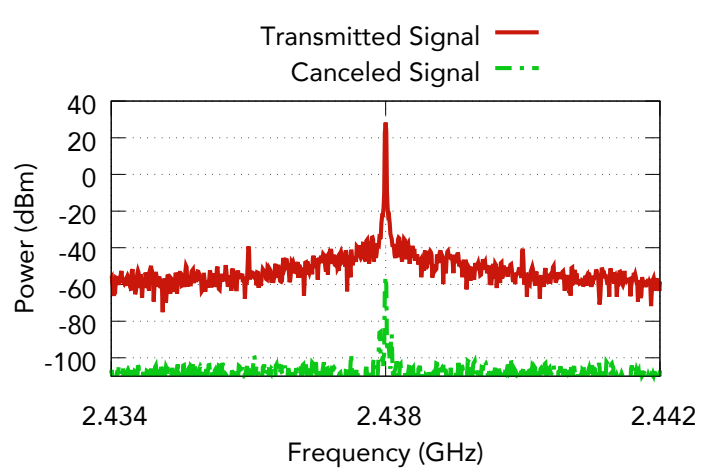

Figure 4: Interference cancellation. Comparison of the transmitted signal to the canceled signal recorded at the receiver demonstrating isolation of approximately $80 \mathrm{~dB}$.

affecting the control voltages, we also operate in the $2.4 \mathrm{GHz}$ ISM band which has other Wi-Fi transmissions. We note that our cancellation hardware can achieve signal cancellation of 80-90 dB, as we describe below. However, better optimized full duplex designs in the literature can achieve higher signal cancellation of up to $100 \mathrm{~dB}$ [28] and can in principle be used to achieve higher communication ranges.

\section{PrintedAnalytics Range Evaluation}

We next evaluate both interference cancellation techniques described above in a typical office environment.

Single Antenna Cancellation. We begin by evaluating our single antenna full duplex setup. We connect our USRP transmitter to a power amplifier and connect the amplifier output to our bi-directional coupler. We connect the through port of the bi-directional coupler to a $3 \mathrm{dBi}$ monopole antenna and use the forward and reverse coupled ports as described above for interference cancellation. To power and control the phase shifter and attenuator we use a bench-top DC power supply. In order to determine the optimal attenuation a and phase shift $\phi$, we begin by transmitting and measuring the received power. Next, we set the attenuation for the reference signal to approximately match the received signal. We then increment the phase starting at $0^{\circ}$. We then adjust the control voltage in coarse increments until we find a minimum, and then do the same for the amplitude. We repeat this process of adjusting the amplitude and phase in successively finer increments until the interference from the transmitter reaches a minimum steady state. Fig. 4 compares power of the transmitted signal which is $28 \mathrm{dbm}$ to the canceled signal, which is $-60 \mathrm{dbm}$. The canceled signal is now much weaker results in the lower receiver noise floor shown in the plot. The figure shows that with the bi-directional coupler we achieve $78 \mathrm{~dB}$ cancellation. This allows us to decode the weak backscatter signals from the 3D printed objects. Note that based on the two-way path loss for backscatter systems this results in a $3 \mathrm{~m}$ diameter coverage.

Bi-Static Cancellation The above implementation leverages $30 \mathrm{~dB}$ of isolation built into the directional coupler, however to further extend the range we also test a bi-static setup. To test this we place our transmitter and receiver antennas $4 \mathrm{~m}$ apart on opposite sides of an office style room. We connect the output of the USRP to a power splitter and connect one end 


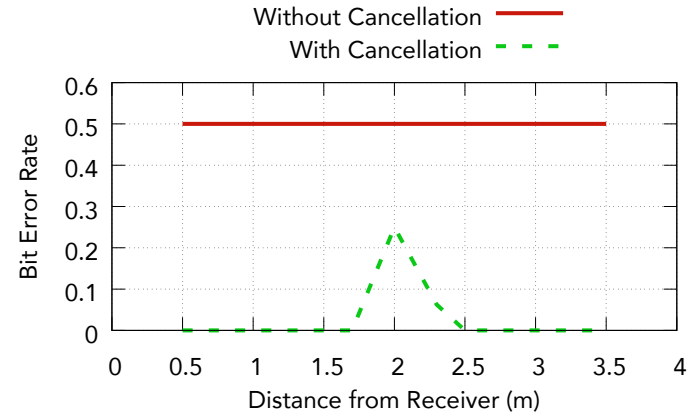

Figure 5: Bit Error Rate (BER). The percentage of incorrectly decoded bits in our bi-static setup when the $3 \mathrm{D}$ printed object is placed at different distances from the receiver. The transmitter is at $4 \mathrm{~m}$, resulting in the symmetric performance characteristic of bi-static backscatter systems. Without cancellation, the BER is $50 \%$.

to a power amplifier which outputs a signal at $20 \mathrm{dBm}$ to the transmit antenna. The second output of the receiver is used as input to the attenuator and phase shifter, and combined with the raw received signal to produce the canceled output. In this setup, we can leverage the free space path loss instead of the directional coupler which attenuates the transmitted signals by $54 \mathrm{~dB}$. We then use our cancellation to further reduce this to a total of approximately $90 \mathrm{~dB}$ isolation resulting in coverage across the $4 \mathrm{~m}$ room.

To more systematically evaluate the above range numbers with 3D printed objects, we evaluate this bi-static deployment by measuring the bit error rate (BER) of the backscattered signal from a 3D printed object for different levels of cancellation. To do this consistently at different locations, we use a rack gear to turn the circular gear to a fixed rotation in our switch mechanism resulting in a standard transmission length. The gear is designed to transmit a total of 20 bits per experiment. We vary the distance between the $3 \mathrm{D}$ printed object while the transmitter and receiver are separated by $4 \mathrm{~m}$ placed on opposite sides of the room. Fig. 5 shows the BER values as function for distance from the RF source.

The plot shows that at low levels of cancellation, the path loss to and from the switch makes the backscattered signal weaker than the transmitted signal and results in many decoding errors past a particular range. Further, the BER is the worse when the backscatter tag is equi-distant from the transmitter and receiver. This is because the backscatter signal deteriorates as a function of $(d 1+d 2)_{2}$, where $\mathrm{d} 1$ and $\mathrm{d} 2$ are the distances from the backscatter object to the transmitter and receiver respectively. The above value is minimized when $d_{1}$ is equal to $d_{2}$, resulting in a worse BER when the backscatter device is equidistant. We also note that without interference cancellation, the BER was consistently close $50 \%$ across the whole range. This is expected because the backscatter signal is significantly weaker at these ranges and cannot be decoded without cancellation. However, at $80 \mathrm{~dB}$ cancellation, the BER values demonstrate a range of $4 \mathrm{~m}$ across the room.

\section{DESIGN OF PRINTEDANALYTICS SENSORS}

Here, we describe our second contribution, enriched bidirectional sensor designs and our third contribution, a new mechanical approach for storing historical information to achieve

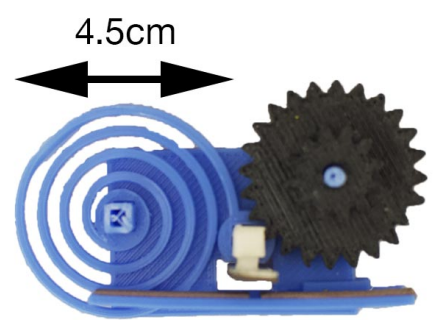

Figure 6: Uni-directional backscatter switch from prior work [24].

analytic sensing even when out of range. Additionally, we discuss the signal processing algorithm used to detect signal peaks caused by actuation of our sensors.

\section{Enriched Printed Backscatter Sensor Designs}

As is common in much prior work (e.g., [24, 43]), a basic capability such as a switch or linear actuation, once achieved, can enable sensing of higher-level signals such as the amount of detergent being poured [24]. Switch actuation is a minimum requirement for rich sensing of mechanical device use, such as actuation of an e-NABLE prosthetic arm or pill bottle.

However, while prior work demonstrates the feasibility of mechanical backscatter with 3D printers [24], the authors failed to successfully construct a true switch. Their initial experiments with a cantilevered approach were not sufficiently robust due in part to material choices (the entire lever bar was printed in conductive material), and they eventually settled on a spring-based design that takes up a large amount of space and is uni-directional as shown in Fig. 6. In contrast, we present a compact, robust and effective cantilever approach that can sense bidirectional motion. Our design uses a rectangular piece of material which is anchored at one end while the other is left free to flex upward or downward as shown in Fig. 7. This structure has a number of advantages as it is simple to design and print, occupies a small area, and has a symmetric response when moving upward or downward. Designing a cantilever structure with backscatter however requires carefully understanding the physics and mechanics as well as the limitations of 3D printers. We analyze these below and present a cantilever structure that matches the performance of prior spring designs while supporting bidirectional motion.

Specifically, the force required to press the spring is determined by the spring constant $k$ and its displacement. Considering the displacement should be roughly the same for either structure, we begin by determining the spring constant of the spiral spring structure and design a cantilever with similar specifications. The spring constant for a spiral spring [5] is given by $\frac{\pi E w t^{3}}{0.00216 L}$ and the spring constant for a rectangular cantilever beam [40] is given by $\frac{E w t^{3}}{4 L^{3}}$, where $E$ represents the Young's modulus of the material, and $w, t$, and $L$ are the width, thickness and length of the beam respectively. We assume a Young's modulus of 3.5 GPa for PLA [3]. While both the length and thickness vary cubicly, we vary the length to achieve the desired results due to fabrication concerns. The typical nozzle diameter of commercial desktop 3D printers is $0.4 \mathrm{~mm}$ which limits at least one axis of the design. Addi- 


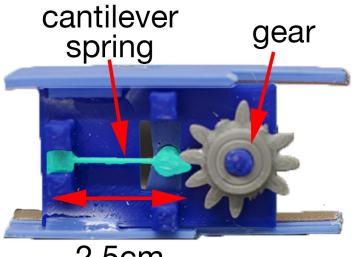

$2.5 \mathrm{~cm}$

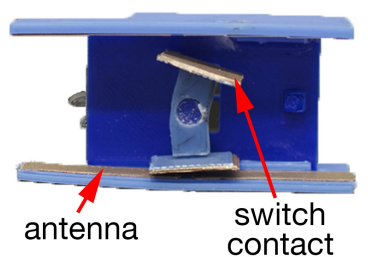

(a) Non-contact state.
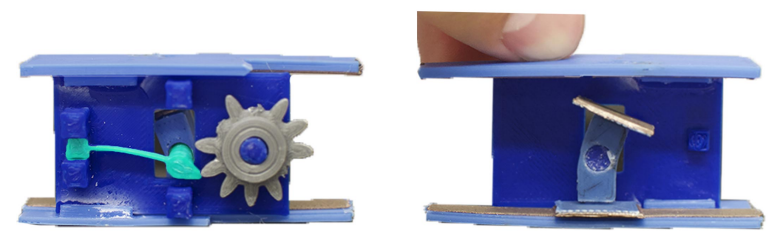

(b) Making contact with bottom antenna.
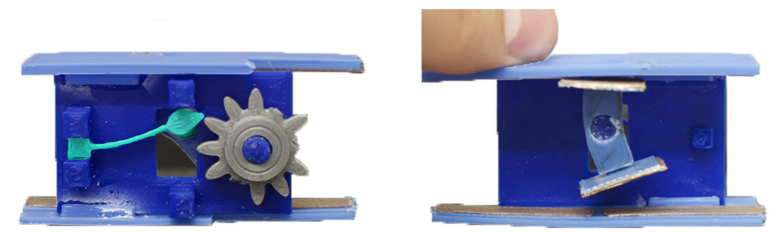

(c) Making contact with top antenna.

Figure 7: Our bi-directional 3D printed backscatter switch. The design has two antennas on either side of the switch.

tionally, while many printers can achieve thin layer heights of 100-200 $\mu \mathrm{m}$, such thin designs tend to be fragile and inconsistent. Based on this, we choose a width of $w=0.76 \mathrm{~mm}$, a thickness of $t=1.88 \mathrm{~mm}$ and length of $L=27.74 \mathrm{~mm}$, achieving $k=207 \mathrm{Nm}^{-1}$ which is similar to the prior spring design, which had $k=171 \mathrm{Nm}^{-1}$. The resulting cantilever spring structure consumes an area of $0.21 \mathrm{~cm}^{2}$ compared to the spiral spring design which requires $19.21 \mathrm{~cm}^{2}$. Using this structure the spring no longer dominates the area of the switch assembly.

We use a gear to actuate the switch as it can easily be coupled to a variety of other mechanical components. Therefore, in addition to having a structure at the end to attach the conductive switch contact, the spring should also easily interact with the gear when it is turned in either direction. To address this we make the tip in the shape of an involute gear tooth to match the profile of the driving gear, as shown in Fig. 7. To anchor the structure we include a $4 \mathrm{~mm}$ cube at one end which is press fit into a $2 \mathrm{~mm}$ thick plastic base. Additionally, to prevent the switch from flexing upward, we print a $1 \mathrm{~mm}$ thick sheet of plastic to place over the top of the structure.

\section{Binary Sensing}

The most basic capability we can enable with backscatter is binary sensing. Each time the backscatter switch is pressed, it transmits information in the form of a changing reflection to which the receiver can detect as a change in amplitude and phase. The switch therefore becomes the primitive for sending any binary data. For example, when a gear rotates past the cantilever structure described above, it presses the switch multiple times in sequence effectively transmitting a sequence of "bits". In an application such as an insulin pen, we can translate the linear motion of a button press attached to a rack gear to turn a circular gear which actuates the switch.

\section{Rotation sensing}

We can easily extend this basic idea to achieve rotational sensing. This capability is useful in a variety of contexts such as detecting when a user unscrews a bottle cap. Because the backscatter switch is actuated by the turning of a gear, we can detect the angle of rotation by the number of times it presses the switch. The gear teeth quantize the number of possible positions and therefore determine the resolution of angular sensing. For example, a gear with 36 teeth would actuate the switch for each rotation of 10 degrees.

One important issue in implementing rotation sensing is resolution. While any two gears will result in some backscattered signal, the gear ratio between the driving gear attached to the arm joint and the backscatter gear will impact the amount of rotation that can be measured. We can increase the angular granularity at which we can track rotation by increasing the gear ratio, or attaching a larger gear with more teeth at item being rotated, and a smaller one on the backscatter mechanism. Intuitively, moving the large gear for even a small fraction of the angle will cause multiple rotations of the smaller backscatter gear. This is most likely to be a relevant consideration for applications where rotation is confined to 360 degrees or less.

\section{Sensing Bi-directional Motion}

In addition to sensing the amount of rotation, we would ideally like to know its direction as well. For applications like the e-NABLE prosthetics we would like to detect whether a user opened or closed the hand. We achieve this by leveraging our cantilever spring which can move in either direction, and place an antenna on either side of it. To determine the direction, we use a gear patterned with an asymmetric code embedded in the sequence of teeth that produces a different pattern of reflections depending on the direction of rotation. We experiment with a variety of methods such as modulating the length and width of gear teeth, however we find that these methods impede the motion of the gear and its interaction with the switch. Instead, inserting small gaps between strings of gear teeth is both effective and simple to fabricate. Gaps such as the omission of a single tooth introduce a time delay between successive presses of the switch. We can see this in the backscattered signal as gaps in time between the successive amplitude peaks produced by consecutive gear teeth moving past the switch. An example of an asymmetric code on a gear is one which begins with a string of six teeth, followed by strings of four and three respectively. We stack an additional gear with a full set of teeth on top of the coded gear to ensure it can still interface with the system. By looking at this order we can determine which direction the gear is rotating.

\section{Anywhere Analytics: Storing Analytics on the Object}

While room-scale sensing is an important improvement, many of the 3D printed objects we would most like to track the use of may travel from room to room (e.g., [1, 10,35]). An ability to track analytics outside the range of the RF source 


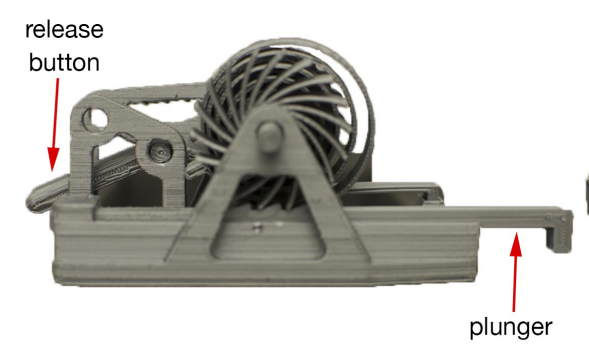

(a) Front view

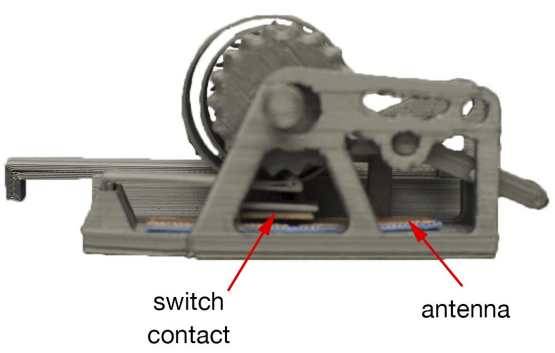

(b) Back view

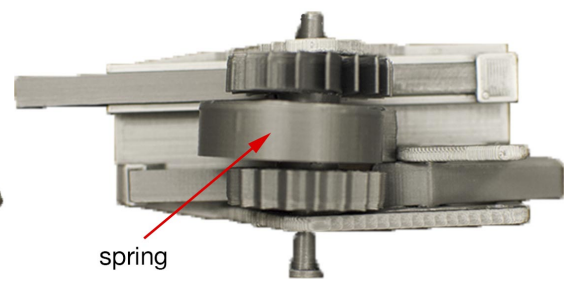

(c) Top view

Figure 8: Supporting Anywhere analytics. We use a 3D printed ratchet system that can hold state about usage by pushing the plunger. The stored data can be later transmitted wirelessly by pushing the release button.

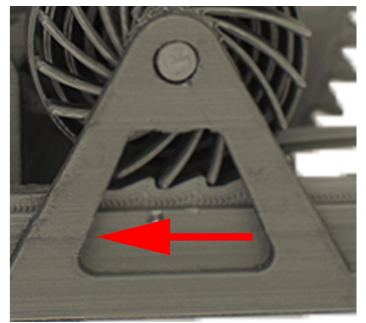

(a) Depressing plunger.

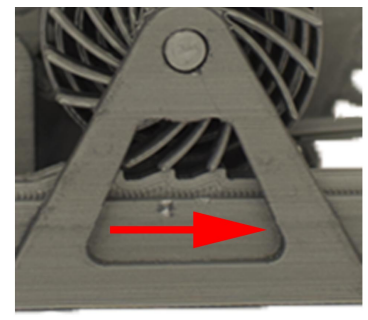

(b) Releasing plunger.
Figure 9: Ratchet mechanism when plunger is in motion.

and receiver would make analytics much more feasible. Here we present a data storage mechanism for 3D printed objects that allows them to store user input and transmit it back to a wireless receiver when brought within range.

To do this, we need two basic capabilities: a mechanism that can hold state to which we "write" data, and a method of releasing this mechanism to "read" back the data wirelessly. For the first capability, we can exploit mechanical designs that can only move in a single direction, such as a ratchet. Because of its ability to only turn in a single direction, a ratchet allows us to "store" the amount we have rotated it. Additionally, to read back the amount of rotation, we need an energy storage mechanism to drive the ratchet back to its original position. We implement this by coupling the rotational motion to a spiral spring. Then, when the ratchet is released by the press of a button, it allows the spring to rotate back to its original state and outputs the stored rotation. In order to communicate this wirelessly, we simply couple the spring rotation to our printed backscatter communication system.

As shown in Fig 9a, our prototype takes input from a plunger which is pressed by the user. This interacts with the ratchet which consists of a spiral shaped mechanism similar to a gear but with angled teeth that interact with gear teeth on the plunger angled in the opposite direction. When the plunger is pressed down, it presses the edges of the spiral gear and rotates it counterclockwise; however the plunger can move upward with minimal resistance. In this case the angled teeth align with the spiral gear and can slide past as shown in Fig 9b. The spiral ratchet mechanism is coupled to a spring attached to the same axle which coils up as it rotates counterclockwise. Additionally, the same axle is also attached to a gear mounted above a cantilever structure with a switch contact. The motion of this gear is limited to moving in one direction by release button therefore preventing the attached spring from unwinding; however when this button is pressed, it releases the spring, allowing the gear to rotate freely. The rotating gear presses the switch contact down as in the previous switch design described above, creating a backscattered signal. While this setup creates the desired motion transfer from the uncoiling spring to the switch, it unwinds too fast to consistently press the switch. In order to release the energy from the spring at a controlled rate, we also include a gearbox. The gearbox consists of a system of 5 involute spur gears on two axles with a 3:1 ratio.

\section{Receiver algorithm}

We implement our receiver by using a USRP N210 software defined radio to record the raw radio signals and write custom processing software to decode the data. The primary challenge for decoding the backscattered data is differentiating it from other reflections in the environment as well as user motion.

To address this, we first subtract a moving average from the signal. This acts in effect like a high pass filter preserving the sharp peaks of the backscattered signal while rejecting other low frequency variations in the environment. As a result, we can now clearly see the transmitted bits. We further apply a low pass filter to remove any high frequency noise which may obscure the signal. We decode the final data by simply applying a threshold to count the bits.

\section{Limitations}

Sensing rotation with high resolution introduces a trade off between resolution and form factor, as adding more gear teeth will consume additional space based on the minimum feature size a $3 \mathrm{D}$ printer can fabricate. We can however relax this constraint by using a ratio of gears. For example, in order to keep the switch mechanism itself small and self contained, we could couple its motion to an external gear with a higher number of teeth to achieve the desired resolution.

Additionally, the coding mechanism we introduce for sensing bi-directional motion exposes other potential problems. We assume that users will make continuous motions with relatively 


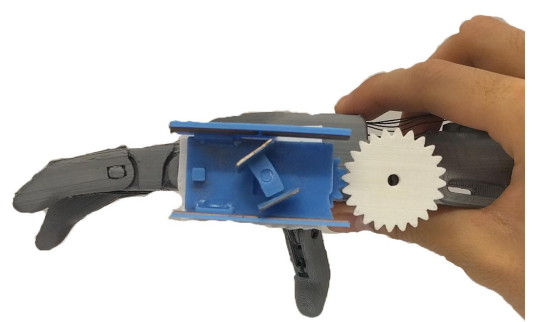

(a) Resting position

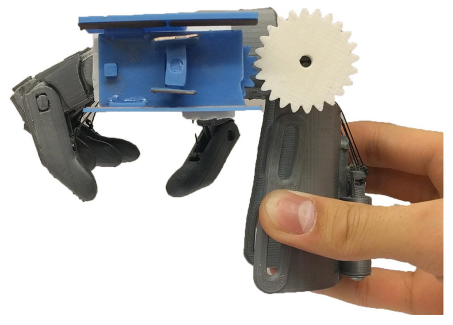

(b) Flexing downwards

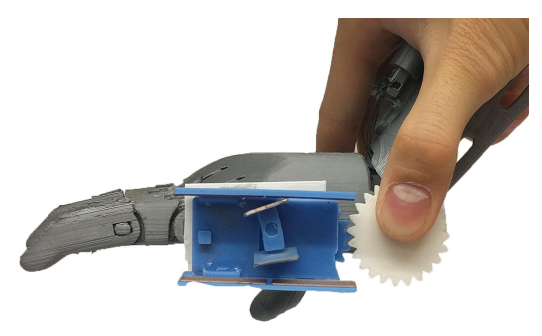

(c) Flexing upwards

Figure 10: e-NABLE hand integrated with our 3D printed backscatter switch.

constant velocities, however a rapidly changing motion could cause a gap between two gear teeth to appear the same as two consecutive gear teeth. To alleviate this challenge, our receiver computes the gear teeth boundaries by comparing adjacent gears. The assumption we make here is that while the signals from the gears can have different durations across all the gears, during consecutive gears, the user motion velocity is relatively similar. The receiver uses this and compares the signals for consecutive gears to address this challenge.

The method we describe above is limited to storing or accumulating a single value and functions like a counter. In addition to only storing a single value, it cannot store information like the time or location associated with the sensed value. The maximum count is limited by how tightly the spring can be coiled, which in our insulin pen prototype corresponds to eight button presses. We design our spiral ratchet assuming the button will be retracted after each press, however an alternative design would be to add more teeth along the surface of the button to measure how much it is depressed. Future designs could improve upon this design by using multiple coupled ratchet gears to store more information, and perhaps look to early mechanical computing machines for inspiration.

Finally, while backscatter can achieve 11 Mbps rates [27], our printed system's rate is limited by the spring switching time of about $20 \mathrm{~ms}$. This approaches human response time and could be improved in the future by changing the spring design.

\section{INTEGRATION WITH APPLICATIONS}

We integrate our backscatter design with both existing and new CAD designs of potential use case applications.

\section{e-NABLE Prosthetic Hand}

The e-NABLE movement is an international group of volunteers 3D printing upper-limb prosthetic devices, primarily for children [1]. The most popular e-NABLE arms are passive, mechanical devices that are actuated by wrist or elbow motion [38]. These devices are frequently mentioned in the

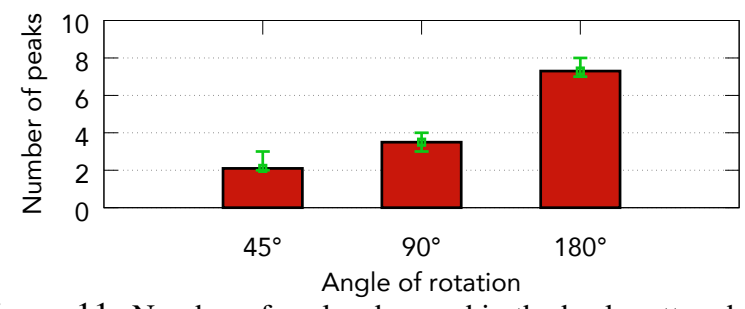

Figure 11: Number of peaks observed in the backscattered signal for different angles of rotation of the e-NABLE hand. media, however important questions about their practical value remain unanswered, because little is known about what happens to those arms after they are delivered [22]. Unfortunately, the abandonment rates for assistive technology are as high as $25-75 \%[39,42,54]$. Capturing data about their use could provide an important picture of how these devices are used.

What is Tracked: Rotation Angle and Direction: eNABLE hands are opened and closed using a linear actuator that operates similar to a tendon. The e-NABLE prosthetic is designed with a joint that aligns with a user's wrist and uses a system of strings to control the motion of the hand. Specifically, as a user bends their wrist, it tightens the strings which pulls the fingers closed allowing the user to grasp an object. As shown in Fig. 10, we instrumented an e-NABLE Phoenix Hand [7] to track the amount of contraction and opening of the fingers, as represented by the angle and direction of rotation of the wrist (Fig. 11). In addition to simply logging when users grasp objects with the hand, tracking the angle in the digital domain also allows us to replay and recreate the exact motion. This opens the potential to replay the motion on a robotic arm for further study, and presents an attractive use case as a controller even for users without prosthetics.

Implementation Approach: We instrument the wrist joint, which controls the prosthetics' motion with a patterned gear, which in turn drives the backscatter switch. We experimented with multiple resolutions. If both gears have the same number of teeth (a gear ratio of $1: 1$ ), then for the maximum $90^{\circ}$ wrist motion in one direction, we will only rotate through $\frac{1}{4}$ of the gear's teeth, sending an equal number of backscattered bits and limiting the angular resolution. We implemented a 12 tooth gear driven by either another 12 tooth gear for a 1:1 ratio, and a 24 tooth gear for a $2: 1$ ratio. We attached the larger gear with more teeth at the wrist, and the smaller one on the backscatter mechanism. To track the direction of rotation, we simply use a gear with an asymmetric pattern as described above. Moving the wrist upward produces a distinct backscatter signal, whereas moving it downward produces the signal in reverse. Fig. 12 shows the backscattered signal when we move the wrist downward by $45^{\circ}, 90^{\circ}$, and $180^{\circ}$. For the $1: 1$ configuration we see 1,3 , or 6 bits for each of the angles respectively due to the gear's minimum resolution of $30^{\circ}$. The 2:1 plots however double the number of bits.

We evaluated the accuracy of our sensor using repeat motions of $45^{\circ}, 90^{\circ}$ and $180^{\circ} 10$ times at a distance of approximately $1 \mathrm{~m}$ from our transmitter and receiver. We performed each of these motions manually with varying speeds to reflect real- 


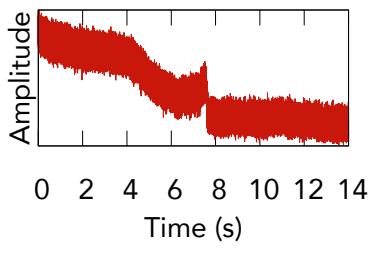

(a) $45^{\circ}$

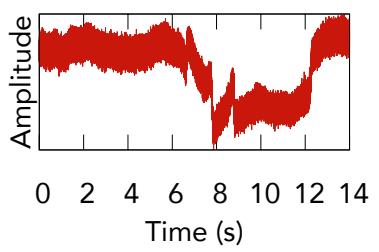

(c) $90^{\circ}$

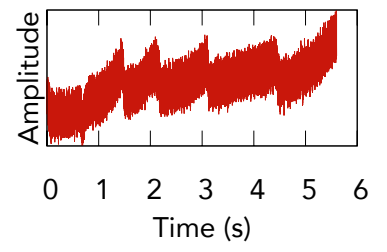

(e) $180^{\circ}$

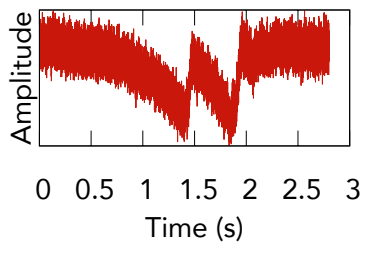

(b) $45^{\circ}$

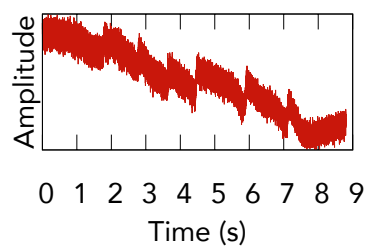

(d) $90^{\circ}$

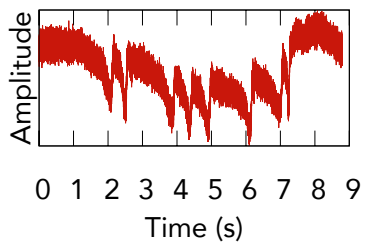

(f) $180^{\circ}$
Figure 12: Raw signal when using the e-NABLE arm (Left) 1:1 gear ratio (Right) 2:1 gear ratio.

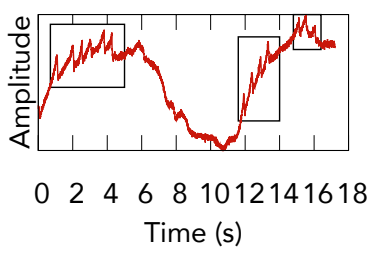

(a) Clockwise signal

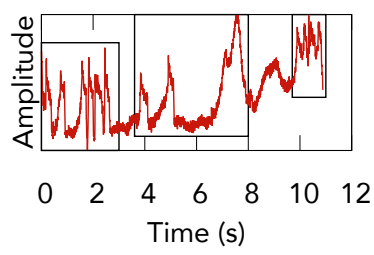

(b) Counterclockwise signal
Figure 13: Pill bottle wireless patterns. We use an asymmetric gear with patterns of consecutive gear teeth each separated by a space. A clockwise rotation shown on the left sends six, four, and three bits, while a counterclockwise rotation sends six, three, and four bits.

world application scenarios. Fig. 11 plots the number of peaks detected in the signal by our algorithm. As can be seen, the error bars are small, with a maximum difference for each angle of 1 bit. Note that the spaces in our gear, used to determine direction, add some ambiguity at these points. Thus, the potential error is 1 tooth, which for a 24 tooth gear corresponds to $15^{\circ}$. This shows that overall our technique is robust and invariant to the speeds at which the wrist flexes.

\section{Pill Bottle}

The number of older adults with chronic conditions, especially complex multi-disease conditions, is increasing [23]. For these adults, medication taking is an important part of disease management [14]. However, medication adherence is low [29], and in many cases unintentionally so [18]. Feedback can increase the consistency of medication taking as well as self efficacy [31]. An inexpensive, customizable solution like a

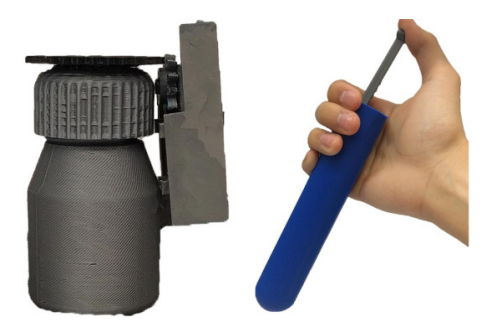

Figure 14: Application prototypes. (a) shows our pill bottle prototype and (b) shows our insulin pen case. For the latter, the user pushes the plunger to use the insulin pen, which in turn uses our ratchet mechanism to store the usage data.

printed pill bottle (a popular assistive technology use of 3D printers [10]) that senses pill taking can help with this problem.

What is Tracked: Rotational Direction: We track when the top of the pill bottle is rotated to open or close the bottle, which is the common metric used by many existing electronic and battery-powered smart pill bottles [2]. We instrument a battery-free bottle to track the direction of rotation.

Implementation Approach: Fig. 14 shows a 3D printed pill bottle outfitted with backscatter. We attach a gear flat on the lid of the bottle. The backscatter gear, switch and antenna could be included within the bottle, however for illustration we mount our prototype vertically on the outside. As the cap is rotated, the gear attached to it drives the patterned gear in the backscatter mechanism that creates the reflected signal.

We can distinguish between the orthogonal opening and closing motions of the bottle using the same patterned gear mechanism as the e-NABLE arm. However, the bottle introduces an additional complexity of the gear moving upward as a user unscrews the bottle cap. To address this we increase the thickness of the gear. This design ensures that some part of it remains in contact with the setup throughout its full cycle of upward or downward motion. Since the cap has to be rotated through multiple revolutions to be opened and closed, there was no need for a gear reduction. We plot the signal as the bottle is opened in a counterclockwise motion in Fig. 13(a). The plot shows the patterns of consecutive gear teeth each separated by a space, specifically six, four, and three in this order. This sequence corresponds to a counterclockwise rotation to open the bottle. In contrast Fig. 13(b) shows the result of clockwise motion in which the same pattern is transmitted in reverse order indicating the direction. Additionally, the plots shows there are gaps in the backscatter signal as the user readjusts their hand between each $180^{\circ}$ rotation of the cover.

We note that the accuracy of this measurement is limited only by the RF performance of the system; as long as the user makes a clockwise or counter-clockwise motion our mechanism will create the backscattered signal pattern and our ability to detect that then comes down to the number of bit errors we can tolerate. We design our code to be robust to single bit errors using a number of features. First, our gear has three different sections of consisting of at least three consecutive gear teeth each. This means that we could tolerate 2 or more errors for any of these. Additionally, so long as we can determine 


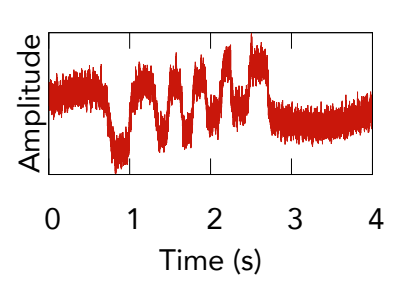

(a)

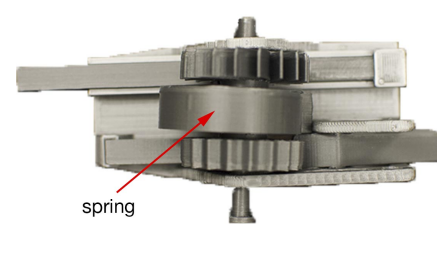

(b)

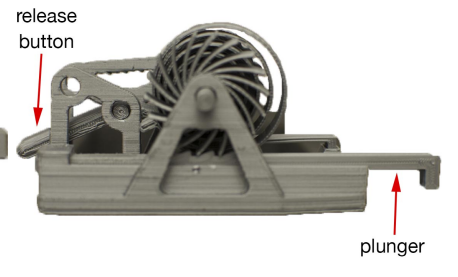

(c)

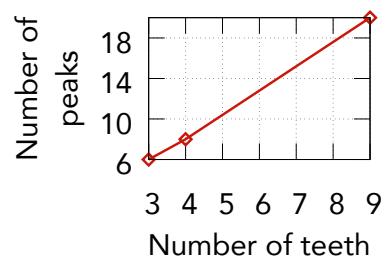

(d)

Figure 15: $(a, b, c)$ Raw signal when pushing down the plunger of the insulin pen by different number of teeth. (d) Relation between number of teeth depressed and number of peaks in the backscattered signal.

the relative size differences between these sequences, this is enough to find the order and therefore the direction.

\section{Insulin Pen}

As with other diseases, the prevalence of diabetes is increasing, and expected to reach 366 million worldwide by 2030 [55]. One common focus of diabetes self-tracking apps is keeping track of insulin use [13]. An electronic-free and battery-free smart insulin pen is attractive because it addresses many of the cost and power issues with existing solutions.

What is Tracked: History (\# Times Insulin is Dispensed): Our prototype is designed to record and store the number of times a user dispenses insulin, and transmit this information when in range. The device consists of a plunger, as described above, which the user would press to dispense insulin. When depressed, a linear actuator moves the gear teeth on the ratchet, which coils the spring, which is held in position by a release button. Upon pressing the release button, when the user reenters range of the wireless receiver, the spring uncoils and the attached gear sends the backscattered message. While the time of the insulin use can be monitoring when administered in wireless range, a limitation of our prototype is that it can record the insulin usage quality but not the time of its usage, when the pen is used outside the wireless range. Additionally, our design does not automatically upload the data, it requires the user to manually press the button when they return within wireless range.

Implementation Approach: This application only requires unidirectional motion, allowing for a simple switch design: we can adjust the number of bits transmitted by adjusting the size of the gear or by coupling a large gear attached to the spring to a smaller gear driving the switch. Fig. 15(a) shows the signal when the plunger is pressed 3 times, it backscatters 6 bits of data. Similarly Fig. 15(b) and (c) show the plot of the signal when the button is depressed 4 and 9 times respectively. Additionally, Fig. 15(d) shows the number of backscattered bits per button press. We note that this relationship remains linear until the last press, after which the spring is fully coiled and the plunger can only be depressed part way.

\section{CONCLUSIONS AND FUTURE WORK}

We have demonstrated a compact, viable approach for sensing the use of a variety of 3D printed objects. Our sensor design is well suited to sensing linear or rotational mechanical actions.
Our sensing depends on backscatter, and an important contribution of this paper is an algorithm and approach for increasing backscatter range to room scale sensing $(4 \mathrm{~m})$. While this on its own is a significant contribution, we also introduce a novel mechanical design capable of storing historical information about the number of object uses that occurs outside the range of our sensing system. When the user returns, a simple press of a button can be used to report on this number.

Several opportunities for future work present themselves. First, we intend to explore design opportunities for sensors of physical rather than mechanical motion. For example, it should be possible to use a marble that rolls inside a tube to trigger mechanical changes and sense motion.

Second, an end-to-end design tool that could simplify the integration of backscatter sensing into a variety of objects would be an interesting challenge. Automated mechanism design has been demonstrated in other contexts (e.g., [9]), and a similar optimization-based approach could be of value here.

Third, the mechanical structures presented in this paper create audible acoustic signals which could in principle be used for wireless sensing. Acoustic-based systems however are susceptible to interference from environmental noise including human speech and typically have a much smaller range than RF-based systems. Exploring this tradeoff between RF and acoustic approaches is an interesting future research direction.

Fourth, our ratchet design for storing data requires user intervention when the object is back in radio range. While acceptable for many applications, designing a wireless receiver at the $3 \mathrm{D}$ printed object that can sense the RF signals and automatically backscatter the stored data, without any user intervention, is an interesting future research direction.

Finally, the size of the mechanical components presented in this paper are chosen for ease of prototyping as well as due to current 3D printer resolution limitations. One could reduce the antenna size by using meandered patterns or choosing a higher frequency. Recent MEMS literature achieves $1 \mu \mathrm{m}$ and smaller feature resolution; this shows a path for future miniaturization as printer resolution and materials improve [52].

\section{ACKNOWLEDGMENTS}

We thank the NSF, Sloan foundation and Google Faculty Awards, along with the UW Reality Lab, Facebook, Google, Huawei and the Richard E. Ladner Chair in Accessibility. 


\section{REFERENCES}

1. 2018. Phoenix Hand. (2018). http://enablingthefuture . org/phoenix-hand/.

2. 2018. Pillsy. (2018). https://www.pillsy.com/.

3. 2018. Polylactic Acid (PLA, Polylactide). (2018). https://www .makeitfrom.com/material-properties/ Polylactic-Acid-PLA-Polylactide.

4. 2018. RVA-33. (2018). https://www .minicircuits. com/pdfs/RVA-33+.pdf.

5. 2018. Spring calculation. (2018). http://mitcalc. com/doc/springs/help/en/springs.htm/.

6. 2018. The Friis Equation. (2018). http://www . antenna-theory. com/basics/friis.php.

7. 2018. Unlimbited Phoenix Hand. (2018). http://www. thingiverse.com/thing: 1674320 .

8. Dinesh Bharadia, Kiran Raj Joshi, Manikanta Kotaru, and Sachin Katti. 2015. Backfi: High throughput wifi backscatter. In SIGCOMM'15. ACM.

9. Gaurav Bharaj, Stelian Coros, Bernhard Thomaszewski, James Tompkin, Bernd Bickel, and Hanspeter Pfister. 2015. Computational design of walking automata. In Proceedings of the 14th ACM SIGGRAPH/Eurographics Symposium on Computer Animation. ACM, 93-100.

10. Erin Buehler, Stacy Branham, Abdullah Ali, Jeremy J Chang, Megan Kelly Hofmann, Amy Hurst, and Shaun K Kane. 2015. Sharing is caring: Assistive technology designs on thingiverse. In Proceedings of the 33rd Annual ACM Conference on Human Factors in Computing Systems. ACM, 525-534.

11. Ji-Hyuk Choi, Han Wang, Soong Ju Oh, Taejong Paik, Pil Sung, Jinwoo Sung, Xingchen Ye, Tianshuo Zhao, Benjamin T Diroll, Christopher B Murray, and others. 2016. Exploiting the colloidal nanocrystal library to construct electronic devices. Science 352, 6282 (2016), 205-208.

12. Jung Il Choi, Mayank Jain, Kannan Srinivasan, Phil Levis, and Sachin Katti. Achieving single channel, full duplex wireless communication. In MobiCom'10. ACM.

13. Andrew P Demidowich, Kevin Lu, Ronald Tamler, and Zachary Bloomgarden. 2012. An evaluation of diabetes self-management applications for Android smartphones. Journal of Telemedicine and Telecare 18, 4 (2012), 235-238.

14. Rohan A Elliott. 2006. Problems with medication use in the elderly: an Australian perspective. Journal of Pharmacy Practice and Research 36, 1 (2006), 58-66.

15. Richard Fletcher, Jeremy A Levitan, Joel Rosenberg, and Neil Gershenfeld. 1996. Application of smart materials to wireless ID tags and remote sensors. In MRS Proceedings.

16. Richard R Fletcher and Neil A Gershenfeld. 2000. Remotely interrogated temperature sensors based on magnetic materials. In IEEE Trans. on Magnetics 'OO.
17. Patrick F Flowers, Christopher Reyes, Shengrong Ye, Myung Jun Kim, and Benjamin J Wiley. 2017. 3D printing electronic components and circuits with conductive thermoplastic filament. Additive Manufacturing 18 (2017), 156-163.

18. Abhijit S Gadkari and Colleen A McHorney. 2012. Unintentional non-adherence to chronic prescription medications: how unintentional is it really? BMC Health Services Research 12, 1 (2012), 98.

19. Shyamnath Gollakota and Dina Katabi. Zigzag decoding: combating hidden terminals in wireless networks. In SIGCOMM'08. ACM.

20. Daniel Halperin, Thomas Anderson, and David Wetherall. Taking the sting out of carrier sense: interference cancellation for wireless LANs. In MobiCom'08. ACM.

21. Chris Harrison, Robert Xiao, and Scott Hudson. 2012. Acoustic barcodes: passive, durable and inexpensive notched identification tags. In UIST'12. ACM.

22. Megan Hofmann, Julie Burke, Jon Pearlman, Goeran Fiedler, Andrea Hess, Jon Schull, Scott E Hudson, and Jennifer Mankoff. 2016. Clinical and maker perspectives on the design of assistive technology with rapid prototyping technologies. In Proceedings of the 18th International ACM SIGACCESS Conference on Computers and Accessibility. ACM, 251-256.

23. William W Hung, Joseph S Ross, Kenneth S Boockvar, and Albert L Siu. 2011. Recent trends in chronic disease, impairment and disability among older adults in the United States. BMC Geriatrics 11, 1 (2011), 47.

24. Vikram Iyer, Justin Chan, and Shyamnath Gollakota. 2017. 3D printing wireless connected objects. ACM Transactions on Graphics (TOG) 36, 6 (2017), 242.

25. Vikram Iyer, Vamsi Talla, Bryce Kellogg, Shyamnath Gollakota, and Joshua Smith. 2016. Inter-technology backscatter: Towards internet connectivity for implanted devices. In SIGCOMM'16. ACM.

26. Bryce Kellogg, Aaron Parks, Shyamnath Gollakota, Joshua R. Smith, and David Wetherall. 2014. Wi-fi Backscatter: Internet Connectivity for RF-powered Devices. In SIGCOMM'14. ACM.

27. Bryce Kellogg, Vamsi Talla, Shyamnath Gollakota, and Joshua R Smith. 2016. Passive Wi-Fi: bringing low power to Wi-Fi transmissions. In NSDI '16.

28. Dani Korpi, Mikko Heino, Clemens Icheln, Katsuyuki Haneda, and Mikko Valkama. 2017. Compact inband full-duplex relays with beyond $100 \mathrm{~dB}$ self-interference suppression: Enabling techniques and field measurements. IEEE Transactions on Antennas and Propagation 65, 2 (2017), 960-965.

29. Sunil Kripalani, Xiaomei Yao, and R Brian Haynes. 2007. Interventions to enhance medication adherence in chronic medical conditions: a systematic review. Archives of Internal Medicine 167, 6 (2007), 540-549. 
30. Gierad Laput, Eric Brockmeyer, Scott E Hudson, and Chris Harrison. 2015. Acoustruments: Passive, acoustically-driven, interactive controls for handheld devices. In $C H I$ '15. ACM.

31. Matthew L Lee and Anind K Dey. 2014. Real-time feedback for improving medication taking. In CHI' 14 . ACM, 2259-2268.

32. Dingzeyu Li, David I.W. Levin, Wojciech Matusik, and Changxi Zheng. 2016. Acoustic Voxels: Computational Optimization of Modular Acoustic Filters. In SIGGRAPH'16. ACM.

33. Dingzeyu Li, Avinash S Nair, Shree K Nayar, and Changxi Zheng. 2017. AirCode: Unobtrusive Physical Tags for Digital Fabrication. In UIST'17. ACM.

34. Vincent Liu, Aaron Parks, Vamsi Talla, Shyamnath Gollakota, David Wetherall, and Joshua R. Smith. 2013. Ambient Backscatter: Wireless Communication out of Thin Air. In SIGCOMM'13. ACM.

35. Scott E Hudson Megan K Hofmann, Gabriella Han and Jennifer Mankoff. 2018. Greater than the Sum of its PARTs: Expressing and Reusing Design Intent in 3D Models. In CHI'18. ACM, To Appear.

36. Saman Naderiparizi, Mehrdad Hessar, Vamsi Talla, Shyamnath Gollakota, and Joshua R Smith. Towards Battery-Free HD Video Streaming. In NSDI'18.

37. Aaron Parks, Shyamnath Gollakota, and Joshua R Smith. 2014. Turbocharging ambient backscatter communication. In SIGCOMM'14. ACM.

38. Jeremiah Parry-Hill, Patrick C Shih, Jennifer Mankoff, and Daniel Ashbrook. 2017. Understanding volunteer at fabricators: opportunities and challenges in diy-at for others in e-nable. In CHI'17. ACM, 6184-6194.

39. Betsy Phillips and Hongxin Zhao. 1993. Predictors of assistive technology abandonment. Assistive technology 5, 1 (1993), 36-45.

40. Mark A Poggi, Andrew W McFarland, Jonathan S Colton, and Lawrence A Bottomley. 2005. A method for calculating the spring constant of atomic force microscopy cantilevers with a nonrectangular cross section. Analytical chemistry 77, 4 (2005), 1192-1195.

41. Stevan Preradovic, Isaac Balbin, Nemai Chandra Karmakar, and Gerhard F Swiegers. 2009.

Multiresonator-based chipless RFID system for low-cost item tracking. In Microwave Theory and Techniques '09.

42. Marti L Riemer-Reiss and Robbyn R Wacker. 2000. Factors associated with assistive technology discontinuance among individuals with disabilities. Journal of Rehabilitation 66, 3 (2000), 44.

43. Michael L Rivera, Melissa Moukperian, Daniel Ashbrook, Jennifer Mankoff, and Scott E Hudson. 2017. Stretching the bounds of 3D printing with embedded textiles. In CHI'17. ACM, 497-508.

44. Valkyrie Savage, Sean Follmer, Jingyi Li, and Björn Hartmann. 2015a. Makers' marks: Physical markup for designing and fabricating functional objects. In UIST'15. ACM, 103-108.
45. Valkyrie Savage, Andrew Head, Björn Hartmann, Dan B. Goldman, Gautham Mysore, and Wilmot Li. 2015b. Lamello: Passive Acoustic Sensing for Tangible Input Components. In $\mathrm{CHI}$ '15. ACM, New York, NY, USA.

46. Martin Schmitz, Martin Herbers, Niloofar Dezfuli, Sebastian Günther, and Max Mühlhäuser. 2018. Off-Line Sensing: Memorizing Interactions in Passive 3D-Printed Objects. In $\mathrm{CHI}$ '18. ACM, New York, NY, USA.

47. Martin Schmitz, Mohammadreza Khalilbeigi, Matthias Balwierz, Roman Lissermann, Max Mühlhäuser, and Jürgen Steimle. 2015. Capricate: A Fabrication Pipeline to Design and 3D Print Capacitive Touch Sensors for Interactive Objects. In UIST'15. ACM.

48. Andrew Spielberg, Alanson Sample, Scott E Hudson, Jennifer Mankoff, and James McCann. 2016. RapID: A framework for fabricating low-latency interactive objects with RFID tags. In CHI'16. ACM, 5897-5908.

49. Vamsi Talla, Bryce Kellogg, Shyamnath Gollakota, and Joshua R. Smith. 2017. Battery-Free Cellphone. Proc. ACM Interact. Mob. Wearable Ubiquitous Technol. 1, 2, Article 25 (June 2017), 25:1-25:20 pages.

50. Vamsi Talla, Bryce Kellogg, Benjamin Ransford, Saman Naderiparizi, Shyamnath Gollakota, and Joshua R. Smith. 2015. Powering the Next Billion Devices with Wi-fi. In CoNEXT'15.

51. Marynel Vázquez, Eric Brockmeyer, Ruta Desai, Chris Harrison, and Scott E. Hudson. 2015. 3D Printing Pneumatic Device Controls with Variable Activation Force Capabilities. In CHI'15. ACM.

52. Andreas Waldschik and Stephanus Büttgenbach. 2010. Micro gear pump with internal electromagnetic drive. Microsystem Technologies 16, 8 (01 Aug 2010), 1581-1587.

53. Anran Wang, Vikram Iyer, Vamsi Talla, Joshua R. Smith, and Shyamnath Gollakota. 2017. Making Everyday Objects into FM Radio Stations. In NSDI'17.

54. R Wessels, B Dijcks, M Soede, GJ Gelderblom, and L De Witte. 2003. Non-use of provided assistive technology devices, a literature overview. Technology and disability 15, 4 (2003), 231-238.

55. Sarah Wild, Gojka Roglic, Anders Green, Richard Sicree, and Hilary King. 2004. Global prevalence of diabetes: estimates for the year 2000 and projections for 2030 . Diabetes care 27, 5 (2004), 1047-1053.

56. Karl D. D. Willis and Andrew D. Wilson. 2013. InfraStructs: Fabricating Information Inside Physical Objects for Imaging in the Terahertz Region. In SIGGRAPH'13. ACM.

57. Pengyu Zhang, Mohammad Rostami, Pan $\mathrm{Hu}$, and Deepak Ganesan. 2016. Enabling practical backscatter communication for on-body sensors. In SIGCOMM'16. ACM. 